commonly made in certain types of popular music and dance among black Caribbeans, changing norms of relationships in an era where you can "order sex via app", peer pressure, and a normalisation of concurrency on social media, especially among men.

Conclusion Among black Caribbeans, the different types and contexts of concurrent partnerships can have implications for STI prevention. Awareness of being in a concurrent partnership could potentially facilitate uptake of interventions including condom use, partner notification, and reduce the risk of re-infection. In addition, such interventions should address broader sociocultural factors influencing risk behaviour including the impact of media.

\section{P4.22 CROSSING THE BRIDGE: EXPLORING SEXUAL RISK PROFILES OF MEN WHO HAVE SEX WITH MEN ATTENDING A SEX ON PREMISES VENUE AND A PUBLIC SEXUAL HEALTH CLINIC FOR STI SCREENING IN SYDNEY, AUSTRALIA}

Catriona Ooi, David Lewis. Western Sydney Sexual Health Centre, Parramatta, Australia

10.1136/sextrans-2017-053264.519

Introduction In Australia, men who have sex with men (MSM) are targeted to curb the spread of HIV and STIs. 'Non-gay' identifying MSM (NGMSM) may not identify with health messages, impacting knowledge of HIV/STI harm minimisation and result in poorer health-seeking behaviours. NGMSM and other men who have sex with men and women are often hidden to clinical services. These men may act as bridges for HIV/STI transmission to female partners. Novel strategies to reach this group include opportunistic HIV/STI screening at sex on premises venues (SOPV). We compared the demographics, testing and sexual behaviour of MSM attending a SOPV to MSM attending an established sexual health clinic (SHC).

Method A daytime SOPV HIV/STI screening service was conducted 2-3 consecutive days per month from November 2015 for 12 months. All patrons were offered testing. The comparison group were MSM attending a local SHC for screening the week following each SOPV clinic. The SHC operates weekdays with appointment and walk-in options. At both sites, participants consented to provide demographic information, contact details and a brief sexual history. Demographics, sexual behaviour and testing practices were compared between the 2 groups.

Results During the study period 84 men tested at the SOPV and 108 at the SHC. SOPV testers were older (mean age, 48.4 years. vs 34.6 years.; $\mathrm{p}<0.001$ ) and were more likely to have had sex with a female in the past 12 months (49/84, $58 \%$ vs. $19 / 105,18 \% ; \mathrm{p}<0.001)$. Compared with SHC testers, more SOPV testers had never had an HIV test $(23 / 84$, $27 \%$ vs. $12 / 108,11 \% ; \mathrm{p}<0.01)$. In the previous 3 months, $100 \%$ condom use with regular partners was similar in both groups (SOPV 33/84, 39\% vs. SHC 37/105, 35\%; p=0.67).

Conclusion Inconsistent condom use with casual and regular sex partners, combined with higher reported rates of sex with females, may enable SOPV testers to act as bridges for STI/ HIV transmission between MSM and heterosexual populations. Our findings have implications for HIV/STI service provision, contact tracing and local health promotion initiatives.
P4.23 EGOCENTRIC SEXUAL NETWORK CHARACTERISTICS OF MEN WHO HAVE SEX WITH MEN (MSM) AND TRANSGENDER WOMEN (TW) RECENTLY DIAGNOSED WITH SYPHILIS AND/OR GONORRHOEA/CHLAMYDIA (GC/CT) IN LIMA, PERU: NETWORK PATTERNS AS ROADMAPS FOR STI PREVENTION INTERVENTIONS

${ }^{1}$ Cherie S Blair, ${ }^{1}$ Eddy R Segura, ${ }^{2}$ Amaya Perez-Brumer, ${ }^{3}$ Jorge Sanchez, ${ }^{4}$ Javier R Lama, ${ }^{1}$ Jesse L Clark. 'David Geffen School of Medicine At UCLA, Department of Medicine and Program in Global Health, Los Angeles, USA; ${ }^{2}$ Columbia University Mailman School of Public Health, New York, USA; ${ }^{3}$ Universidad Nacional Mayor San Marcos, Lima - Peru; ${ }^{4}$ Asociación Civil Impacta Salud y Educación, Lima - Peru

\subsection{6/sextrans-2017-053264.520}

Introduction Endemic rates of STIs like GC/CT and syphilis in Peru are only partially explained by individual behavioural or biological factors. Characterisation of sexual networks of MSM/TW with syphilis and/or GC/CT can provide critical data to inform prevention efforts.

Methods We enrolled $917 \mathrm{MSM} / \mathrm{TW}$ as screening for 2 STI control trials in Lima, Peru. We surveyed demographics and sexual identity, role, and 30 day network characteristics (number/gender of sexual partners, partner types, and frequency of anal/vaginal intercourse) and tested for syphilis (RPR >1:16) and oral, rectal, or urethral GC/CT (TMA). Differences in egocentric network characteristics were analysed with Chisquare and Kruskal-Wallis tests.

Results Approximately 38.7\% $(\mathrm{n}=355)$ of subjects had a new STI diagnosis (Syphilis: 97 [10.6\%]; GC/CT: 161 [17.6\%]; Syphilis-GC/CT Co-infection: 49 [5.3\%]). MSM/TW with GC/ CT were younger (median age+IQR: 25 [22-30]) than those with syphilis (28 [23-34]) or no STI (28 [24-35]). STI-negative subjects were more likely to identify their sexual role as activo (insertive; $24.8 \%$ ) than men with syphilis $(10.8 \%)$ or GC/CT (14.7\%). MSM/TW with GC/CT reported greater median numbers of all partners (3 [2-5]) and of casual male or transgender partners $(2[0-4])$ than those with syphilis $(2[1-5]$ and 1 [0-2], respectively) or no STI (2 [1-3] and 1 [0-2]). Both $\mathrm{GC} / \mathrm{CT}$ and syphilis were associated with the number of partnerships involving receptive anal intercourse (RAI; 2 [1-4] and 2 [1-13]) compared with STI-uninfected subjects (1 [0-2]). No differences were noted in the number of partnerships with condomless RAI or number of female partners.

Conclusion Egocentric network characteristics of MSM/TW with GC/CT and/or syphilis demonstrated progressive increases in network size, number of casual partners, and frequency of RAI when comparing no infection vs. syphilis vs. GC/CT +/syphilis co-infection. Detailed understanding of network patterns, along with individual and partnership characteristics, will inform public health responses to HIV/STIs among Latin American MS.

\section{P4.24 SWIPE LEFT ON DISEASE: THE EFFECTS OF CONTEXTUAL FACTORS AND DATING APP USAGE ON SEXUAL RISK BEHAVIOURSAMONG YOUNG ADULTS}

Chloe Chan. New York University, New York, USA

\subsection{6/sextrans-2017-053264.521}

Introduction With the rise of social media and smartphones, millennials increasingly report meeting their romantic partners through mobile dating apps. Yet there is concern that this may also be the reason behind increases in sexually 